\title{
Within reach but not so reachable: Obstacles matter in visual perception of distances
}

\author{
Nicolas Morgado • Édouard Gentaz • Éric Guinet • \\ François Osiurak • Richard Palluel-Germain
}

Published online: 14 December 2012

(C) Psychonomic Society, Inc. 2012

\begin{abstract}
A large number of studies have shown that effort influences the visual perception of reaching distance. These studies have mainly focused on the effects of reach-relevant properties of the body and of the objects that people intend to reach. However, any influence of the reach-relevant properties of the surrounding environment remains still speculative. We investigated this topic in terms of the role of obstacle width in perceiving distances. Participants had to estimate the straightline distance to a cylinder located just behind a transparent barrier of varying width. The results showed that participants perceived the straight-line distance to the cylinder as being longer when they intended to grasp the cylinder by reaching around a wide transparent barrier rather than by reaching around narrower ones. Interestingly, this effect might be due to the anticipated effort involved in reaching. Together, our results show that reach-relevant properties of the surrounding environment influence perceived distances, thereby supporting an embodied view of the visual perception of space.
\end{abstract}

Keywords Distance perception · Peripersonal space · Economy of action · Effort · Perception-action link

N. Morgado · É. Gentaz • É. Guinet · R. Palluel-Germain Laboratoire de Psychologie et Neurocognition (CNRS), Université de Grenoble, Grenoble, France

N. Morgado

e-mail: nicolas.morgado@upmf-grenoble.fr

F. Osiurak

Laboratoire d'Étude des Mécanismes Cognitifs (EA 3082),

Université Lyon 2, Lyon, France

R. Palluel-Germain $(\bowtie)$

Laboratoire de Psychologie et Neurocognition,

Université Pierre-Mendès-France, 1251 Avenue Centrale, BP 47,

38040 Grenoble Cedex 9, France

e-mail: richard.palluel@upmf-grenoble.fr
When you reach around a water bottle to grasp a soda placed just behind it, the distance that you have to cover depends on the bottle width. Even if variations of the bottle width imply different reaching distances, the distance in a straight line between your hand and the soda (i.e., the Euclidean distance) remains identical. According to modular approaches (e.g., Pylyshyn, 1999), the intended reaching distance should not influence your visual perception of the Euclidean distance, since effort or intentions are not supposed to influence vision. Researchers using these approaches have argued that any influence of action capabilities must necessarily operate at the response rather than the perceptual stage (Durgin et al., 2009; Woods, Philbeck, \& Danoff, 2009).

In contrast, an embodied view (e.g., Glenberg, 2010) of perception, namely the economy-of-action account (Proffitt, 2006; for somewhat similar views, see also Coello \& Delevoye-Turrell, 2007; Jackson \& Cormack, 2007), posits that the effort associated with intended actions influences the visual perception of space. The rationale of this account is that sensory motor systems have evolved under evolutionary pressures that promote minimizing such action costs as energy expenditure. For instance, reducing the arm's reach with wrist weights (Lourenco \& Longo, 2009) or making an object harder to grasp by manipulating the orientation of its handle (Linkenauger, Witt, Stefanucci, Bakdash, \& Proffitt, 2009) leads people to perceive this object as being farther away. Conversely, people perceive an object beyond reach as being closer when they intend to reach it with a baton that extends their arm's reach than when they intend to reach without this tool (Witt, Proffitt, \& Epstein, 2005). These results suggest that modifications of the reach-relevant properties of a body (i.e., an arm's reach) and of the objects that people intend to reach (i.e., handle orientation) influence perceived Euclidean distances. However, the argument that these results can be generalized to the influence of reach-relevant properties of the surrounding environment remains speculative. 
Studies about the influence of tool use on perceived distance have provided preliminary insights into this question. Tools are objects from the surrounding environment that can improve one's ability to act, influencing space perception even in situations in which these objects are not explicitly defined as tools (Osiurak, Morgado, \& Palluel-Germain, 2012). However, such effects of tool use probably rely on extension of the arm's reach that is associated with an updating of the body schema (e.g., Cardinali et al., 2009) - that is, a reach-relevant property of the body, but not a property of the environment per se. Unlike tools, obstacles are part of the surrounding environment, so they are not supposed to directly affect body or target properties. Instead, obstacles mediate the relationship between the body and the target. For this reason, obstacles are particularly relevant for studying the influence of reach-relevant properties of the surrounding environment without modifying the body and target properties. In line with the economy-of-action account, we expected (and observed) that intending to grasp a cylinder by reaching around a transparent barrier leads participants to estimate the Euclidean distance to this cylinder differently, depending on the barrier width.

\section{Method}

\section{Participants}

Twenty right-handed undergraduates (19 female, one male; $M_{\text {age }}=21.75, S D_{\text {age }}=3.34$ ) from the University of Grenoble took part in this experiment for course credit. The handedness of the participants was assessed by self-report and with the Edinburgh Handedness Survey (Oldfield, 1971; $M=85.47$, $S D=16.26$ ). The participants had normal or corrected-tonormal vision, as indicated by self-report. The present study was conducted in accordance with the Declaration of Helsinki and with the understanding and the written consent of each participant. It was approved by the local ethics committee of the LPNC (CNRS and the University of Grenoble).

\section{Apparatus and procedure}

The participants sat approximately $5 \mathrm{~cm}$ away from the edge of a rectangular table (length $144 \mathrm{~cm}$, width $125 \mathrm{~cm}$, height $77 \mathrm{~cm})$. They had to estimate the Euclidean distance $(43,40$, 37 , or $34 \mathrm{~cm}$ ) between their right forefinger and a plastic cylinder (height $9 \mathrm{~cm}$, diameter $3.2 \mathrm{~cm}$ ) by a visualmatching task (for similar measures, see Osiurak et al., 2012; Witt et al., 2005). Using the arrows from a keyboard with their left hand, they adjusted the distance between the right forefinger, kept on a reference point, and a comparison point projected onto the table until it matched the Euclidean distance between their finger and the cylinder (Fig. 1). The reference point and the cylinder were aligned on the participant's midsagittal axis. The comparison point was projected upon the table at a $45^{\circ}$ angle from the midsagittal axis in the right or left hemifield of the participant. The initial distance between the comparison point and the reference point was randomly equal to either $\pm 25 \%$ of the distance between the cylinder and the reference point. The cylinder was presented $10 \mathrm{~cm}$ behind a transparent barrier (height $25 \mathrm{~cm}$ ) that was of variable width (wide $30 \mathrm{~cm}$, medium $20 \mathrm{~cm}$, or narrow $10 \mathrm{~cm}$ ). The transparent barrier allowed participants to clearly see the cylinder but was intended to increase the anticipated reaching effort, depending on the barrier's width. It is important to note that before each distance estimation, participants had to imagine a reach-to-grasp movement to the cylinder. This was done because some studies had shown that the ability to perform an action influences perceived distance when people intend to carry out this action (Witt et al., 2005) and that imagined actions influence perceived distances in the same way (Witt \& Proffitt, 2008). In order to reduce between-subjects variability in the imagined movements, participants actually performed this movement five times before the visual-matching task. More precisely, on another table, they reached around a transparent barrier (height $25 \mathrm{~cm}$, width $15 \mathrm{~cm}$ ) so as to grasp a cylinder presented $23 \mathrm{~cm}$ from a constant initial position. This movement was executed without leaning forward and with the shoulders against the back of the chair. Moreover, to avoid merely priming effort, the barrier width and distance used during this task allowed participants to easily reach and grasp the cylinder with minimal reaching effort.

After this motor task, participants performed the visualmatching task described previously. They first performed four training trials randomly selected from among the 12 distance-width pairs. Then they completed two estimations for each of these pairs, one with the comparison point in their right and one with the comparison point in their left hemifield. These 24 test trials were presented randomly. At the end of each trial, participants masked their eyes with their hands to allow the experimenter to adjust the apparatus for the next trial. To prevent the use of proprioceptive and kinesthetic cues of perceived distances provided by actual movements, the participants never reached over the table during the 28 distance estimations. Cues projected onto the table allowed the experimenter to install the cylinder with the appropriate barrier width at the appropriate distance.

After this visual-matching task, the experimenter interviewed the participants to determine whether they suspected the goal of the experiment using a postexperimental questionnaire. ${ }^{1}$ Finally, participants also rated their anticipated

\footnotetext{
${ }^{1}$ The experimenter asked two questions to the participants: (1) In your opinion, what hypothesis was tested in this study? (2) Do you think that some aspects of the experiment could have influenced your responses? If so, what were these aspects?
} 
Fig. 1 Schematic representation of the visual-matching task. Participants had to put their right forefinger on the reference point (R) throughout the visualmatching task. Then they had to estimate the Euclidean distance between that finger and the cylinder by a visual-matching task. Using the arrows from a keyboard with their left hand, they adjusted the distance between the reference point and a comparison point $(\mathrm{C})$ projected onto the table until that comparison matched the Euclidean distance between their finger and the cylinder. The cylinder was presented $10 \mathrm{~cm}$ behind a transparent barrier (height $25 \mathrm{~cm}$ ) of variable width (wide $30 \mathrm{~cm}$, medium $20 \mathrm{~cm}$, or narrow $10 \mathrm{~cm}$ )

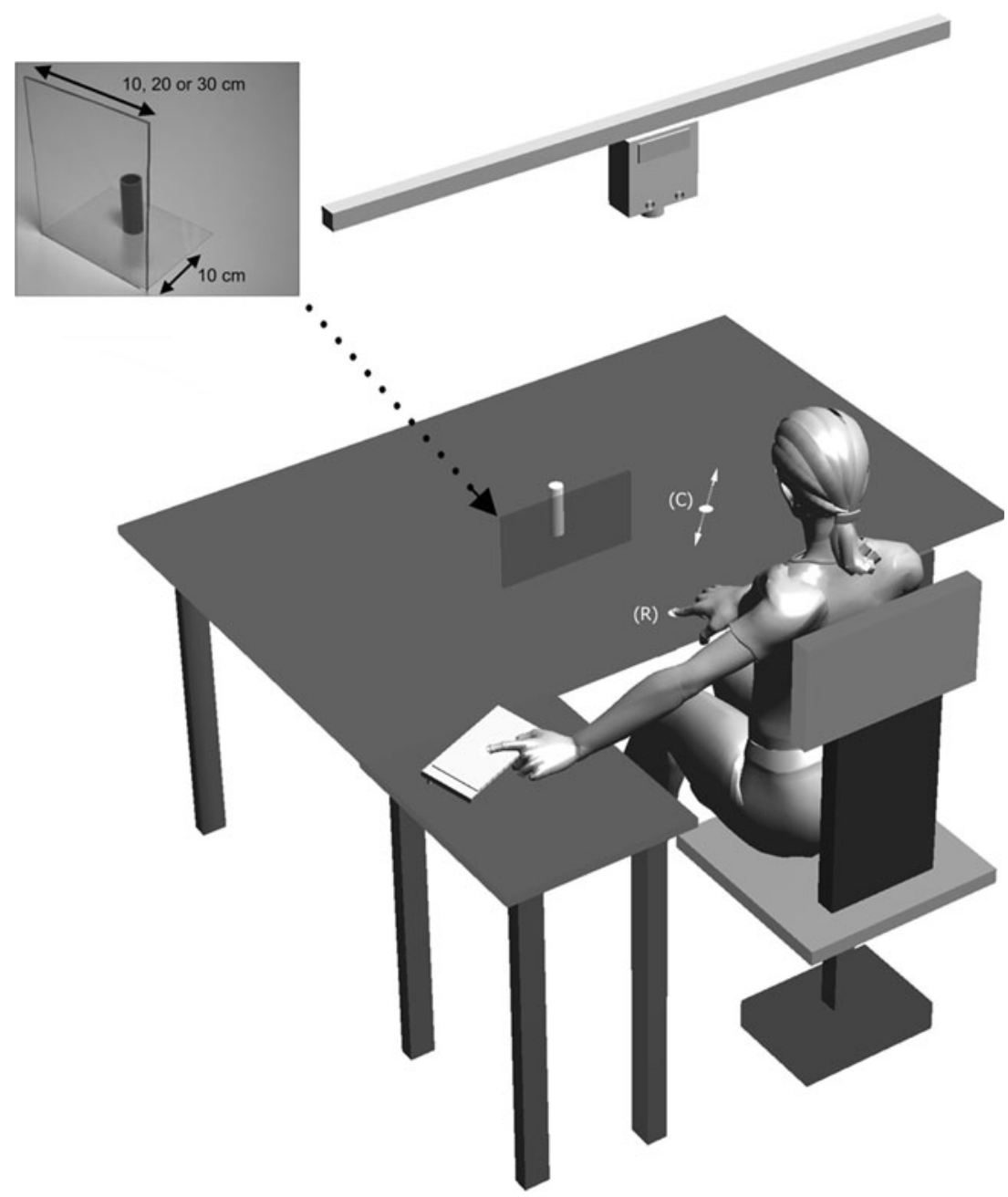

reaching effort for each barrier width on a 4-point scale (1, no effort, to 4, strong effort). For this effort manipulation check, the cylinder was randomly presented with each of the three barrier widths $24 \mathrm{~cm}$ from the reference point. In addition, since the perceived and actual sizes of the body influence space perception (Linkenauger, Witt, \& Proffitt, 2011; Stefanucci \& Geuss, 2009; Van der Hoort, Guterstam, \& Ehrsson, 2011), the experimenter also recorded the perceived and actual lengths of the right and left arms of each participant. The arm length estimations were recorded following a visual-matching task used by Linkenauger, Witt, Bakdash, Stefanucci, and Proffitt (2009) in a counterbalanced order. For each of their arms, the participants had to estimate the distance between the protrusion of their shoulder and the tip of their forefinger. Then, the experimenter measured this distance in order to obtain the actual length of each arm. The effort manipulation check and the measure of the actual and perceived arm's lengths were done after the postexperimental questionnaire. This aimed to ensure that the postexperimental questionnaire reflects as much as possible the suspicion resulting from the visual-matching task and not any potentially produced by these additional measures.

\section{Results}

According to the two questions asked to the participants about our hypothesis, none of the participants indicated a suspicion that we were testing the effect of reaching constraints on the visual perception of distances. We conducted a two-way analysis of variance (ANOVA) with Actual Distance $(43,40,37$, and $34 \mathrm{~cm})$ and Barrier Width (wide $30 \mathrm{~cm}$, medium $20 \mathrm{~cm}$, or narrow $10 \mathrm{~cm}$ ) as within-subjects factors, and Perceived Euclidean Distance as a dependent variable. To avoid the frequent problem of the sphericity assumption, we separated this ANOVA into a group of orthogonal contrasts with one degree of freedom (Judd, McClelland, \& Ryan, 2009). ${ }^{2}$ This principle was also

\footnotetext{
${ }^{2}$ An omnibus ANOVA was also performed and yielded similar results for the effects of actual distance, $F(3,57)=348.26, p<.001, \eta^{2}=.95$, and barrier width, $F(2,38)=3.34, p=.02, \eta^{2}=.19$. However, the interaction between actual distance and barrier width was not significant with this omnibus ANOVA, $F(6,114)=1.16, p=.33, \eta^{2}=.06$, whereas it was marginal when tested with the contrast analysis, which is known to be a more powerful and conservative test than omnibus ANOVA (Judd et al., 2009).
} 
used for the subsequent analyses. The main effect of the actual distance was significant, as indicated by the significant linear contrast, $F(1,19)=847.79, p<.001, \eta^{2}=.98$, and the nonsignificant quadratic and cubic contrasts, tested together, $F(1,38)=2.92, p=.096, \eta^{2}=.07$. More interestingly, we also found a significant effect of the barrier width, as indicated by the significant linear contrast, $F(1,19)=$ 4.43, $p=.049, \eta^{2}=.19$, and the nonsignificant quadratic contrast, $F(1,19)=3.81, p=.066, \eta^{2}=.17$. Supplemental analyses using Bonferroni correction revealed that the distance overestimation was significant between the wide $(M=$ $41.62 \mathrm{~cm}, S D=2.86)$ and narrow $(M=40.42 \mathrm{~cm}, S D=$ 2.69) barriers, $p=.026$, and was marginal between the wide and medium ones $(M=40.63 \mathrm{~cm}, S D=2.51), p=.088$. However, the distance estimations for the medium and narrow barriers were not significantly different, $p>9$. The interaction between the barrier width and the actual distance was marginal, as indicated by the marginal linear contrast, $F(1,19)=4.23, p=.054, \eta^{2}=.18$, and the nonsignificant residual contrast, $F(1,95)=1.39, p=.24, \eta^{2}=.01$. This was partly due to the fact that the effect of the barrier width was not significant for the shortest distance, $F(1,19)=0.32, p=$ $.58, \eta^{2}=.02$, whereas it was significant for the three longest distances, $p \mathrm{~s}<.05$ (Fig. 2).

Concerning the effort manipulation check, we conducted a one-way ANOVA with Barrier Width as a within-subjects factor and the rating of the anticipated reaching effort as a dependent variable. We observed that the anticipated reaching effort increased significantly with the barrier width, $F(1$, $19)=69.28, p<.001, \eta^{2}=.78$. However, this increase was significantly greater between the medium and wide barriers $(M=1.2, S D=0.77)$ than between the narrow and medium ones $(M=0.7, S D=0.66), F(1,19)=5, p=.038, \eta^{2}=.21$. Additionally, we found a significant difference between the perceived lengths of the right $(M=57.27 \mathrm{~cm}, S D=8.33)$ and the left $(M=54.76 \mathrm{~cm}, S D=7.17)$ arms, $F(1,19)=$ $7.04, p=.02, \eta^{2}=.27$. In contrast, the difference was not significant between the actual lengths of the right $(M=$ $68.12 \mathrm{~cm}, S D=3.39)$ and left $(M=68.19 \mathrm{~cm}, S D=3.53)$ arms, $F(1,19)=0.06, p=.8, \eta^{2}=.003$. Interestingly, this
Fig. 2 Perceived distance as a function of actual distance and barrier width. Error bars denote standard errors of the means, corrected for between-subjects variability (Cousineau, 2005)

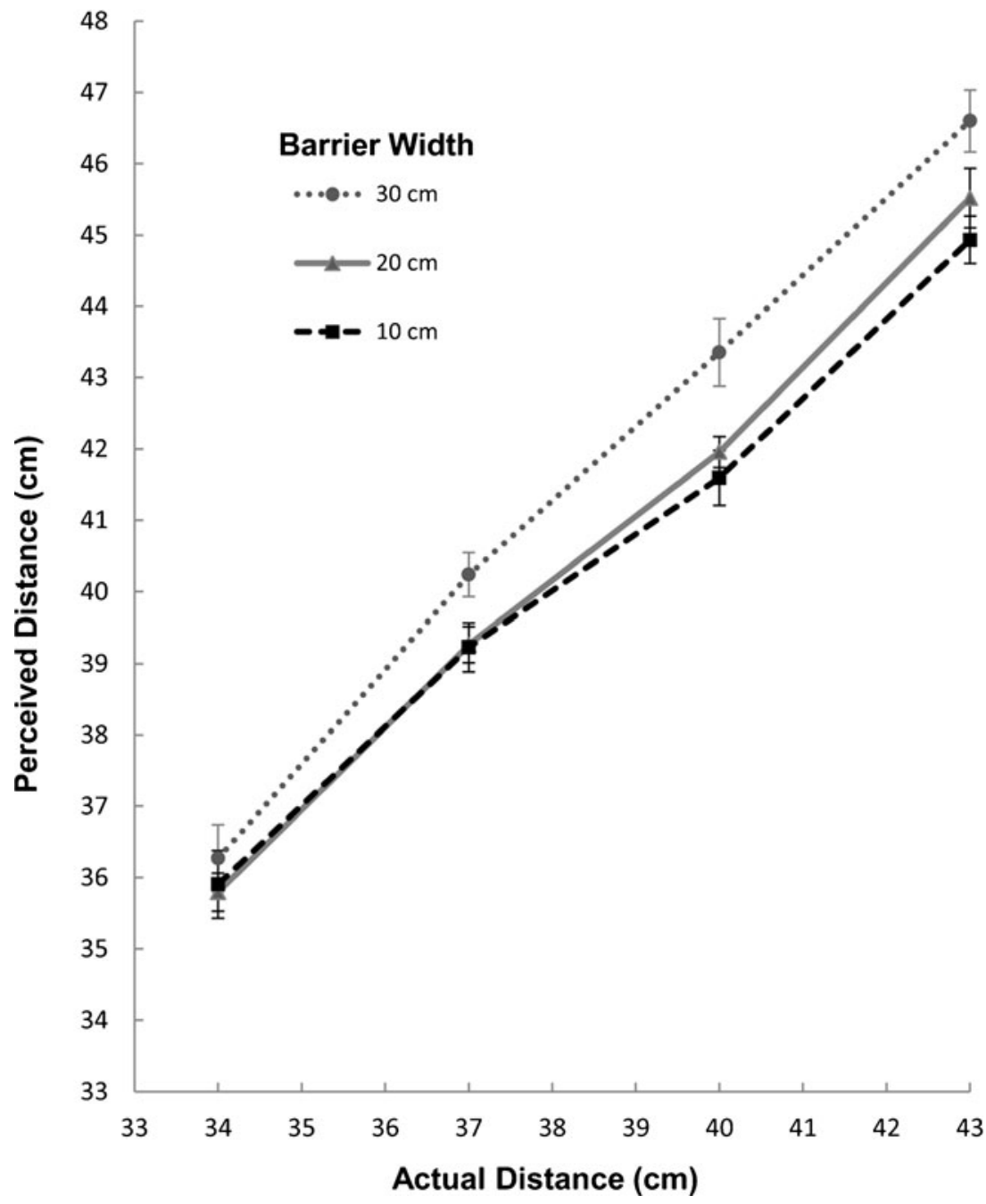


asymmetry in perceived arm length is consistent with those found by Linkenauger, Witt, Bakdash, et al. (2009). However entering perceived or actual right arm length as a covariate in the analysis did not significantly modify the results reported above concerning the influence of barrier width on perceived distances.

\section{Discussion}

In the present study, participants perceived longer Euclidean distances to a cylinder in the presence of a wide barrier than in the presence of medium and narrow ones. As suggested by the effort manipulation check analysis, this might reflect that the difference in the anticipated reaching efforts between the wide barrier and the two others was greater than that between the medium and narrow barriers. However, further studies will have to confirm this interpretation using a more subtle measure of anticipated effort (e.g., Rosenbaum \& Gaydos, 2008). In contrast, one could argue that our results might be explained in terms of demand characteristics. Against such an explanation, it could be argued that the postexperimental questionnaire would have allowed us to detect participants who suspected our hypothesis. Moreover, a difference in the perceived distance between the medium and narrow barriers would have been observed, and we should not have observed a marginal interaction between barrier width and actual distance. In spite of these arguments, we agree that the potential implications of demand characteristics in such experiments remains an important concern (Durgin et al., 2009). Notably, the use of implicit manipulations of effort, the use of indirect measures of perceived distances, and the use of a different type of postexperimental questionnaire might be relevant to deal with this concern in future studies. The absence of a difference in perceived distance between the medium and narrow barriers also rules out an interpretation in terms of distance segmentation produced by the barrier, as had been shown in previous studies of environmental effects on distance perception (e.g., Nasar, 1983; Witt, Stefanucci, Riener, \& Proffitt, 2007).

Consistent with the economy-of-action account, the marginal interaction between the actual distance and the barrier width could reflect that the effort required to reach around the wide barrier increases with the actual distance. Such an interaction effect was also observed by Lessard, Linkenauger, and Proffitt (2009), who showed that increasing the physical constraints of an intended action influences distance perception merely when constraints substantially affect the action capabilities. Further studies will be needed to directly test this hypothesis. In addition, our results corroborate previous studies on motor control that have indicated that the biomechanical costs associated with going around or above obstacles play an important role in motor planning for reaching (Cohen, Biddle, \& Rosenbaum, 2010) as well as for walking (Patla \&
Rietdyk, 1993). More generally, as was stated by Sparrow and Newell (1998), the planning and performing of adaptive actions seem to be functions of an organism's propensity to minimize energy expenditure regarding the task, environment, and organism constraints on an action. Consistent with this statement, perceptual effects such as the one found in the present study could play an important role in economic action planning (Proffitt, 2006).

Detractors of the economy-of-action account have argued that such variability of space perception is unlikely to occur independently of response bias, since an illusory perception of the environment would be dysfunctional for the adaptive control of action (Durgin, Ruff, \& Russell, 2012). However, recent studies have suggested that such top-down effects of action on perception have an adaptive function by influencing action in return. For instance, Elliott, Vale, Whitaker, and Buckley (2009; see also Witt, Linkenauger, \& Proffitt, 2012) showed that increasing the perceived height of a stair by a visual illusion leads people to adopt a safer stepping strategy in order to avoid tripping. Therefore, perceptual effects resulting from the tendency to minimize action costs might be highly adaptive for action planning through promoting larger safety margins. Conversely, perceptual effects resulting from the overestimation of one's own capabilities might have many detrimental effects through increasing risky behaviors. Luyat, Domino, and Noël (2008) provided a compelling example of such tendencies by observing that older adults tend to overestimate their ability to stay on an inclined surface without falling, suggesting that this perceptual tendency could lead to an increase in falling risks.

Perceived Euclidean distances are influenced by modifications of the reach-relevant properties of the body and of objects that people intend to reach, such as arm's reach (Lourenco \& Longo, 2009; Witt et al., 2005) or handle orientation (Linkenauger, Witt, Stefanucci, et al., 2009), respectively. Here, we observed that obstacle width plays a role in this perception as a reach-relevant property of the surrounding environment. These results provide strong support for the claim that action costs influence visual perception (Proffitt, 2006), which appears to be a more penetrable process than is usually assumed. Of course, further studies will need to determine exactly what cognitive processes underlie the effect observed in the present study. It has been shown that both motor simulation (Witt \& Proffitt, 2008) and visual attention (Cañal-Bruland, Zhu, Van der Kamp, \& Masters, 2011) are implicated in the effects of actions on space perception. How these processes interact with visual perception to produce these effects remains an open issue. Another interesting research perspective consists in extending the rationale of the economy-of-action account to social cognition. Recent evidence has suggested that this perspective is promising, since it has been reported that psychosocial resources and costs influence visual perception (Harber, Yeung, \& Iacovelli, 
2011; Morgado, Muller, Gentaz, \& Palluel-Germain, 2011; Schnall, Harber, Stefanucci, \& Proffitt, 2008; for reviews, see Balcetis \& Lassiter, 2010; Schnall, 2011; Stefanucci, Gagnon, \& Lessard, 2011).

Author Note We thank Arthur Glenberg and the reviewers, for their valuable comments on a previous version of the manuscript, and Pauline Oliver, for her help collecting the data.

\section{References}

Balcetis, E., \& Lassiter, G. D. (2010). Social psychology of visual perception. New York, NY: Psychology Press.

Cañal-Bruland, R., Zhu, F. F., Van der Kamp, J., \& Masters, R. S. W. (2011). Target-directed visual attention is a prerequisite for actionspecific perception. Acta Psychologica, 136, 285-289.

Cardinali, L., Frassinetti, F., Brozzoli, C., Urquizar, C., Roy, A. C., \& Farnè, A. (2009). Tool-use induces morphological updating of the body schema. Current Biology, 19, R478-R479.

Coello, Y., \& Delevoye-Turrell, Y. (2007). Embodiment, spatial categorisation and action. Consciousness and Cognition, 16, 667-683.

Cohen, R. G., Biddle, J. C., \& Rosenbaum, D. A. (2010). Manual obstacle avoidance takes into account visual uncertainty, motor noise, and biomechanical costs. Experimental Brain Research, 201, 587-592.

Cousineau, D. (2005). Confidence intervals in within-subject designs: A simpler solution to Loftus and Masson's method. Tutorial in Quantitative Methods for Psychology, 1, 42-45.

Durgin, F. H., Baird, J. A., Greenburg, M., Russell, R., Shaughnessy, K., \& Waymouth, S. (2009). Who is being deceived? The experimental demands of wearing a backpack. Psychonomic Bulletin \& Review, 16, 964-969. doi:10.3758/PBR.16.5.964

Durgin, F. H., Ruff, A. J., \& Russell, R. C. (2012). Constant enough: On the kinds of perceptual constancy worth having. In G. Hatfield \& S. Allred (Eds.), Visual experience: Sensation, cognition, and constancy (pp. 87-102). Oxford, U.K.: Oxford University Press.

Elliott, D. B., Vale, A., Whitaker, D., \& Buckley, J. G. (2009). Does my step look big in this? A visual illusion leads to safer stepping behaviour. PLoS One, 4(e4577), 2159-2168. doi:10.1371/ journal.pone. 0004577

Glenberg, A. (2010). Embodiment as a unifying perspective for psychology. Wiley Interdisciplinary Reviews: Cognitive Science, 1, 586-596.

Harber, K. D., Yeung, D., \& Iacovelli, A. (2011). Psychosocial resources, threat, and the perception of distance and height: Support for the resources and perception model. Emotion, 11, 1080-1090.

Jackson, R. E., \& Cormack, L. K. (2007). Evolved navigation theory and the descent illusion. Perception \& Psychophysics, 69, 353-362.

Judd, C. M., McClelland, G. H., \& Ryan, C. S. (2009). Data analysis: A model comparison approach. London, U.K.: Routledge.

Lessard, D. A., Linkenauger, S. A., \& Proffitt, D. R. (2009). Look before you leap: Jumping ability affects distance perception. Perception, 38, 1863-1866.

Linkenauger, S. A., Witt, J. K., Bakdash, J. Z., Stefanucci, J. K., \& Proffitt, D. R. (2009a). Asymmetrical body perception: A possible role for neural body representations. Psychological Science, 20, 1373-1380.

Linkenauger, S. A., Witt, J. K., \& Proffitt, D. R. (2011). Taking a handson approach: Apparent grasping ability scales the perception of object size. Journal of Experimental Psychology. Human Perception and Performance, 37, 1432-1441.

Linkenauger, S. A., Witt, J. K., Stefanucci, J. K., Bakdash, J. Z., \& Proffitt, D. R. (2009b). The effects of handedness and reachability on perceived distance. Journal of Experimental Psychology. Human Perception and Performance, 35, 1649-1660.

Lourenco, S. F., \& Longo, M. R. (2009). The plasticity of near space: Evidence for contraction. Cognition, 112, 451-456.

Luyat, M., Domino, D., \& Noël, M. (2008). Surestimer ses capacités peut-il conduire à la chute? Une étude sur la perception des affordances posturales chez la personne âgée. Psychologie \& Neuropsychiatrie du Vieillissement, 6, 287-297.

Morgado, N., Muller, D., Gentaz, E., \& Palluel-Germain, R. (2011). Close to me? The influence of affective closeness on space perception. Perception, 40, 877-879.

Nasar, J. L. (1983). Environmental factors, perceived distance and spatial behavior. Environment and Planning B: Planning and Design, 10, 275-281.

Oldfield, R. C. (1971). The assessment and analysis of handedness: The Edinburgh inventory. Neuropsychologia, 9, 97-113. doi:10.1016/ 0028-3932(71)90067-4

Osiurak, F., Morgado, N., \& Palluel-Germain, R. (2012). Tool use and perceived distance: When unreachable becomes spontaneously reachable. Experimental Brain Research, 218, 331-339.

Patla, A. E., \& Rietdyk, S. (1993). Visual control of limb trajectory over obstacles during locomotion: Effect of obstacle height and width. Gait \& Posture, 1, 45-60.

Proffitt, D. R. (2006). Embodied perception and the economy of action. Perspectives on Psychological Science, 1, 110-122.

Pylyshyn, Z. (1999). Is vision continuous with cognition? The case for cognitive impenetrability of visual perception. The Behavioral and Brain Sciences, 22, 341-365.

Rosenbaum, D. A., \& Gaydos, M. J. (2008). A method for obtaining psychophysical estimates of movement costs. Journal of Motor Behavior, 40, 11-17.

Schnall, S. (2011). Embodiment in affective space: Social influences on the perception of spatial layout. In A. Maas \& T. Schubert (Eds.), Spatial dimensions of social thought (pp. 129-152). Berlin, Germany: Mouton De Gruyter.

Schnall, S., Harber, K. D., Stefanucci, J. K., \& Proffitt, D. R. (2008). Social support and the perception of geographical slant. Journal of Experimental Social Psychology, 44, 1246-1255.

Sparrow, W. A., \& Newell, K. M. (1998). Metabolic energy expenditure and the regulation of movement economy. Psychonomic Bulletin \& Review, 5, 173-196.

Stefanucci, J. K., Gagnon, K. T., \& Lessard, D. A. (2011). Follow your heart: Emotion adaptively influences perception. Social and Personality Psychology Compass, 5, 296-308.

Stefanucci, J. K., \& Geuss, M. N. (2009). Big people, little world: The body influences size perception. Perception, 38, 1782-1795.

Van der Hoort, B., Guterstam, A., \& Ehrsson, H. H. (2011). Being Barbie: The size of one's own body determines the perceived size of the world. PLoS One, 6, e20195. doi:10.1371/journal.pone.0020195

Witt, J. K., Linkenauger, S. A., \& Proffitt, D. R. (2012). Get me out of this slump! Visual illusions improve sports performance. Psychological Science, 23, 397-399.

Witt, J. K., \& Proffitt, D. R. (2008). Action-specific influences on distance perception: A role for motor simulation. Journal of Experimental Psychology. Human Perception and Performance, 34, 1479-1492.

Witt, J. K., Proffitt, D. R., \& Epstein, W. (2005). Tool use affects perceived distance, but only when you intend to use it. Journal of Experimental Psychology. Human Perception and Performance, 31, 880-888.

Witt, J. K., Stefanucci, J. K., Riener, C. R., \& Proffitt, D. R. (2007). Seeing beyond the target: Environmental context affects distance perception. Perception, 36, 1752-1768.

Woods, A. J., Philbeck, J. W., \& Danoff, J. V. (2009). The various perceptions of distance: An alternative view of how effort affects distance judgments. Journal of Experimental Psychology. Human Perception and Performance, 35, 1104-1117. 